\title{
Design and fabrication of novel discrete actuators for microrobotic tasks
}

Hussein Hussein, Ismail Bouhadda*, Abdenbi Mohand-Ousaid, Gilles Bourbon, Patrice Le Moal, Yassine Haddab and Philippe Lutz

Univ. Bourgogne Franche-Comté, Femto-st Institute, Besançon, France

Corresponding author: ismail.bouhadda@femto-st.fr

\begin{abstract}
This paper presents a compliant monolithic multistable actuator which is able to switch its moving part between several stable positions linearly in one dimensional direction. The number of stable positions can be increased by extending the range of displacement of the moving part. The transition in each step of displacement is made to the nearest stable position in the direction of motion. Upward and downward steps are made by a specific sequence of moving, using a bistable module, opening and closing two internal clamps which are actuated by U-shaped electrothermal actuator using three subsystems. The principle and the design of each subsystem in the discrete acruator, fabrication process and experimental results are presented. The fabricated prototypes of the discrete actuator showed a proper functioning. The mean achieved displacement is $120.67 \pm 0.08 \mu \mathrm{m}$ over 12 upward steps with a mean step of $10.06 \mu \mathrm{m}$, which is very close to the designed performance.
\end{abstract}

Keywords: preshaped curved beam, U-shaped electrothermal actuator, multistable module, microfabrication, bulk micromachining.

\section{Introduction}

Digital microrobotics is an emergent branch in microrobotics which consists of avoiding sensors by the use of mechanisms with several stable positions [1-4]. In fact, sensors are generally bulky when used in microstructures and pose some problems such as the noise that increases with respect to the signal for small dimensions, resolution and complicated control laws. The mechanism in digital microrobotics is actuated with discrete actuator that generates several mechanical stable and repeatable positions within its range of motion. This allows relying on the stable states to have confidence in the provided positions without need of sensors after switching from state to the other. The concept of discrete actuator has many advantages in digital microrobotics, it allows obtaining robust and repeatable positionning without measurement systems. Excluding the sensors in digital microrobotics allows simplifying the structure, the connectivity and the control, integrating the microrobot in complicated environments and going further in miniaturization. As described in the literature, 
most of the discrete actuators are based on a bistable mechanism [5-17]. Switching between two states, this mechanism guarantees a mechanical stability of the states in open loop without energy consumption unlike stick-slip [18-21], inertial [22-24] or inchworms actuators [25,26]. This widespread mechanism has been employed to design actuators with several stable positions. Among these mechanisms, we find tristable [27-31], quadristable [32,33] and pentastable designs. In the other hand, few unlimited multistable mechanisms are presented in the literature. Gerson et al. [34] presented a multistable microactuator with a large displacement by combining curved beams and serially connected bistable elements. Following, Chalvet et al. presented the concept of digital microrobot based on parallel distribution of bistable elements [3]. However, those mechnisms are subjected to a trade-off between the stable positions (directely related to the number of bistables) and the dimensions of the mechnism. Due to the combination of bistables, the size of the mechanism increases rapidily. As a consequence, the minuaturization and the control of the mechnism become complex and non-intuitive.

To tackle this limitation, a novel compact discret actuator is presented in this paper. Instead of combining several bistables, we propose here a new mechanism architecture with multi stable positions. This new architecture allows switching linearly its moving part between several stable positions in one dimensional direction. It combines the accuracy and robustness advantages of multi-elementary stable microsystems with the stepping principle which allows the actuator to have more positions by simply extending the range of motion as the case of stepping microactuators. Compared to the above mentioned mechnisms, the proposed actuator is a promising solution that adds discrete positions to the workspace with a smaller footprint while adding many advantages. The main characteristics of the new actuator are summarized in the following:

- Compact design with more discrete positions.

- A stepping principle mechanism.

- Positioning robustness due to the digital concept and the use of stable structures.

- The number of internal actuators are reduced and the control is simplified.

- The stability margin due to the holding forces at each stable position.

- Increased accuracy due to the use of a mechanism to compensate microfabrication tolerances.

- No energy is needed to hold the moving part at rest. 
- Compliant structure which has many advantages such as increased precision and reliability, no friction, reduced wear, avoided backlash, minimized hysteresis and low manufacturing costs.

- Monolithic structure which is an essential requirement to realize microsystems with common microfabrication processes and allows the integration in complicated environments. The device is fabricated on SOI wafers using a deep reactive ion etching (DRIE) based process. The fabricated prototype showed a proper functioning in the experiments with accurate and repeatable steps. The novel actuator can be used for accurate positioning applications in MEMS. It can be integrated in more complex systems for more advanced tasks and the design can be changed for different number of stable positions and different step dimensions.

The principle of the multistable mechanism in the novel actuator is presented in section II. Design of the internal systems and components are presented in section 3. The fabrication process is clarified in section 4 and finally the experimental characterization is presented in section 5 .

\section{Principle of the multistable mechanism}

The multistable actuator allows its moving part to switch between several stable positions in a straight line back and forth. This novel actuator consists of three subsystems (see Fig. 1). Each subsystem is composed of two U-shaped electrothermal actuators and flexible structures. These subsystems ensure the switching and holding of the moving part in stable positions.

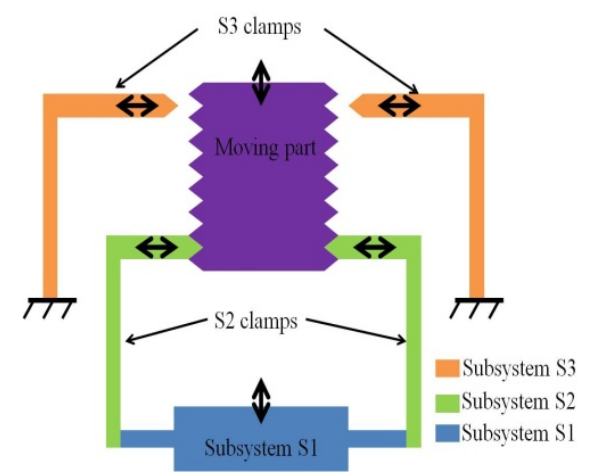

Fig. 1. Illustrative schematic of the multistable actuator.

The three subsystems are respectively further referred to S1, S2 and S3 and both clamps of S2 and S3 to S2 clamp and S3 clamp. The subsystem S1 is a bistable module that allows moving up and down S2 clamp. At the rest configuration of S1, S1 and consequently S2 clamp are at the bottom position. The subsystem S2 allows holding the moving part by passive gearing 
between S2 clamp and the moving part. At the rest configuration of S2, the S2 clamp is closed (see Fig. 1). At active state of S2, the S2 clamp is open (S2 clamp is separated from the moving part). The combination of S1 and S2 allows to drive upwards and downwards the moving part. The subsystem S3 allows holding the moving part when the S2 clamp is open by an active gearing between the S3 clamp and the moving part (S3 clamp is closed). At the rest configuration of S3, the S3 clamp is open (see Fig. 1). The displacement of the moving part is guided on a straight line with a couple of curved beams. It's performed by a sequence of moving, opening and/or closing the clamps with and without the moving part. The sequence order allows making upward steps of the moving part is presented in figure 2.

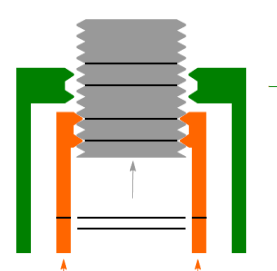

(a)

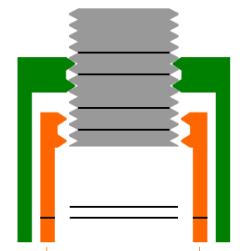

(d)

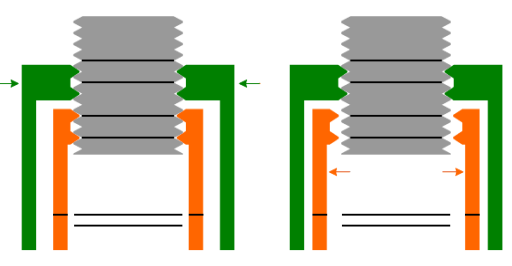

(b)

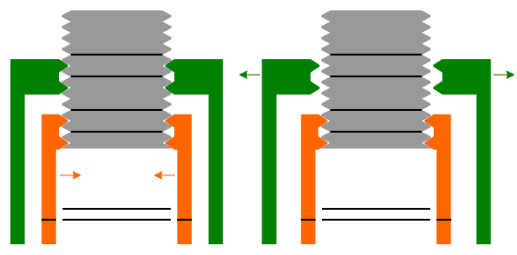

(e)

(f)

Fig. 2. Sequence order to make an upward step. Firstly, S2 clamp move upwards with holding the moving part (a), S3 clamp holds the moving part (b), S2 clamp releases the moving part (c), moves downwards (d) and holds the moving part in a bottom position of S1 (e), finally, S3 clamp releases the moving part (f).

The downward step of the moving part will be achieved by the following sequential order: (b)-(c)-(a)-(e)-(f)-(d). (S3 clamp holds the moving part)-(S2 clamp releases the moving part)(S2 clamp move upwards)- (and closes in the upper position of S1)-( S3 clamp releases the moving part)-( finally, S2 clamp moves downwards with holding the moving part). It worth to notice that during operation at least one of the two clamps holds the moving part.

\section{Design of the multistable actuator}

In this section, the design of the different components and each internal system in the multistable actuator are presented.

\subsection{Subsystem S1}

The subsystem S1 can be classified as a bistable module, which combines the advantages of the digital concept, monolithic structures and compliant mechanisms. As illustrated in Fig. 3, it consists of a shuttle, structurally connected to S2 clamp, which is guided vertically using curved beams, two U-shaped electrothermal actuators and a mechanical stop mechanism. 


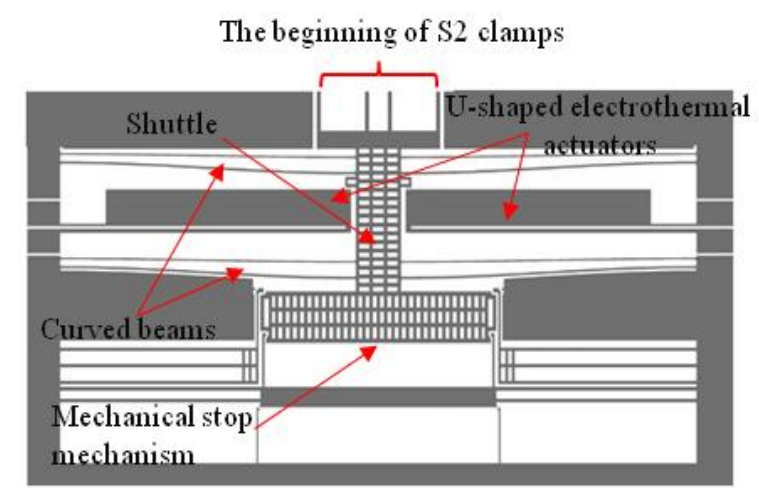

Fig. 3. Schematic draw of subsystem S1 and its different components.

The mechanical stop mechanism that defines stepping distance of the shuttle, consists of two stops and two locks as shown in Fig. 4.

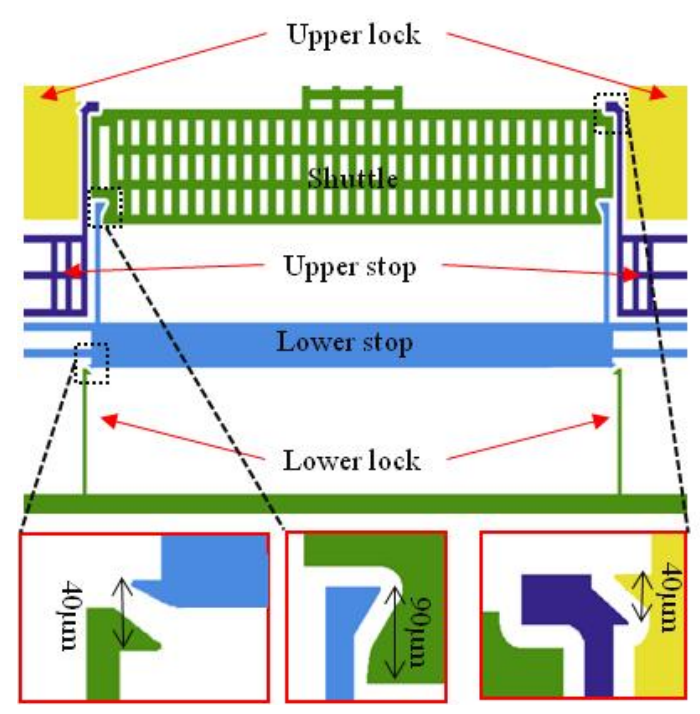

Fig. 4. Schematic draw of the mechanical stop mechanism and its different components.

After the fabrication, an activation phase is required for the mechanical stop mechanism to be operational. This activation phase consists in attaching the lower and the upper stop to the lower and the upper lock respectively (see fig. 5). The shuttle is then placed between the lower and upper stops that define stepping distance $(10 \mu \mathrm{m})$.

After the fabrication, an activation phase is required for the mechanical stop mechanism. This activation, performed only once, allows the mechanical stop mechanism to be operational and define the stepping distance for the shuttle. It consists in attaching, manually using probeheads, the upper stop to the upper lock and respectively the lower stop to the lower lock (figure 5). Once the activation phase is done, the shuttle is placed at the bottom stop position (cf. the middle zoom of the figure 5). The stroke limitation of the shuttle is also defined by the upper stop as indicated in the zoom at the right zoom of the figure 5. 


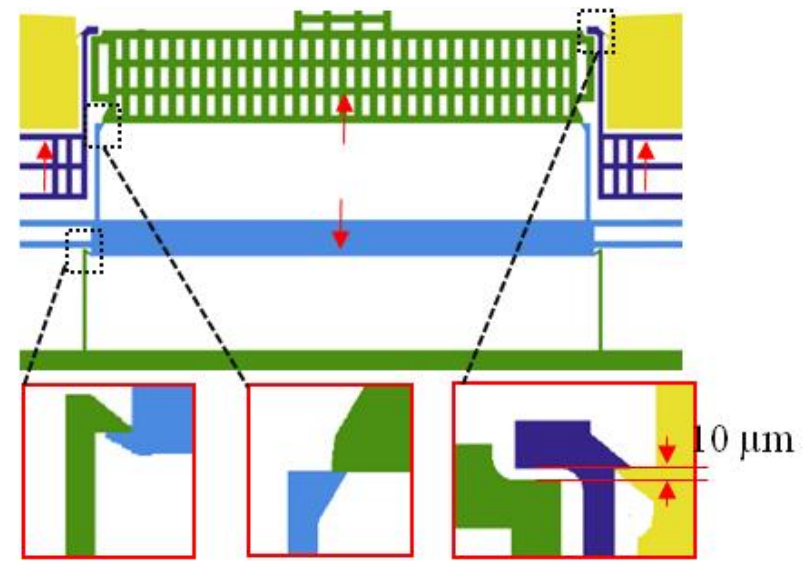

Fig. 5. Activation of the mechanical stop mechanism.

An elastic restoring force of $14.5 \mathrm{mN}$ is obtained when the lower stop is suspended to the lower lock and $7.5 \mathrm{mN}$ for each one of the upper stops when suspended to the upper locks. Therefore, the total forces on the shuttle must not exceed $14.5 \mathrm{mN}$ in the downward direction and $15 \mathrm{mN}$ in the upward direction. In order to improve the robustness of the vertical displacement and to reduce the rotation possibility of the shuttle two couples of curved beams are used. Behavior and design of preshaped curved beams were investigated in [35]. The dimensions of the first and second couples are respectively $1=6.5 \mathrm{~mm} / 6.5 \mathrm{~mm}, \mathrm{~h}=100 \mu \mathrm{m} /$ $30 \mu \mathrm{m}, \mathrm{t}=20 \mu \mathrm{m} / 15 \mu \mathrm{m}$ and $\mathrm{b}=100 \mu \mathrm{m} / 100 \mu \mathrm{m})$, where $1, \mathrm{~h}, \mathrm{t}$ and $\mathrm{b}$ are the length, height, thickness and the depth of the curved beams respectively. These dimensions are chosen to ensure a significant holding force in the first position of the shuttle (at rest) and to avoid high loads on the electrothermal actuators in the second stable position (after a shuttle displacement of $10 \mu \mathrm{m}$ ). The value of the snapping force of the four curved beams together, deduced from FEM simulation is shown in fig. 6. When the shuttle is at rest and after displacement of $10 \mu \mathrm{m}$ the snapping force of the four-curved beam together is $4.4 \mathrm{mN}$ and $4.15 \mathrm{mN}$ respectively.

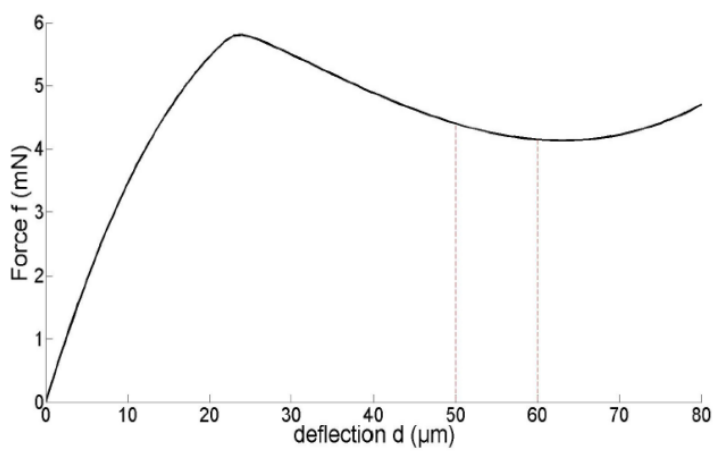

Fig. 6. Snapping force evolution of the curved beams in system 1 during deflection.

The design of subsystem S1 is presented in details in [36].

\subsection{Subsystem S2}

As explained previously, the subsystem S2 allows opening S2 clamp, which is designed to be normally closed after the manual activation phase. It consists of two U-shaped electrothermal 
actuators and S2 clamp structure, which is designed as a gantry in order to ensure horizontal entry and exit of the teeth. Figure 7 shows the drawing of subsystem S2.

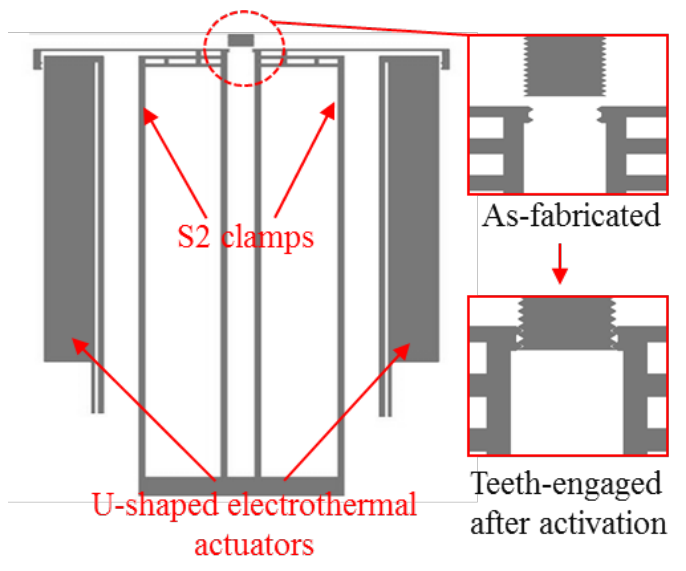

Fig. 7. Drawing of subsystem S2, including a zoom on the teeth as fabricated and after activation.

During the activation phase, the S2 clamp is opened and the moved vertically to be at the lower stop position. The relative distances of the involved parts reported on the figure 5, $40 \mu \mathrm{m}$ and $90 \mu \mathrm{m}$, lead to an upward shuttle displacement of $90 \mu \mathrm{m}-40 \mu \mathrm{m}$, i.e. $50 \mu \mathrm{m}$. This vertically displacement allows the couples of teeth on the head of each side of the clamp become engaged to the first couples of teeth on the moving part. The horizontal distance between the as fabricated and the teeth engaged configurations of S2 clamp is $12 \mu \mathrm{m}$. The horizontal restoring force determined by the $12 \mu \mathrm{m}$ distance and the stiffness of the S2 gantry is about $0.75 \mathrm{mN}$ for each side of the moving part. These forces holding the teeth of the S2 clamp engaged between the teeth of the moving part allows maintaining the moving part at rest and during driving.

During functioning, the clamp S2 must be released the moving part, by the U-shaped electrothermal actuator, at least $10 \mu \mathrm{m}$ (Teeth engagement of clamp and moving part) to ensure no contact between the teeth of S2 clamp and moving part during the vertical displacement of S2 clamps, thanks to the system 1. In order to ensure a proper functioning in the multistable actuator, the security distance opening of S2 clamp is fixed at least $15 \mu \mathrm{m}$ (see fig. 8). The security distance remains small since combined with the stiffness of gantry, it determines the required driving force of the electrothermal actuators for opening the S2 clamp.

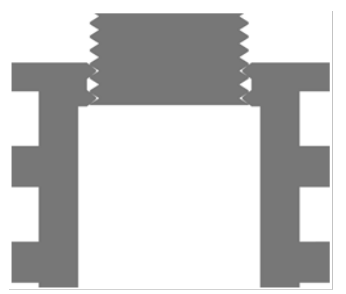

(a)

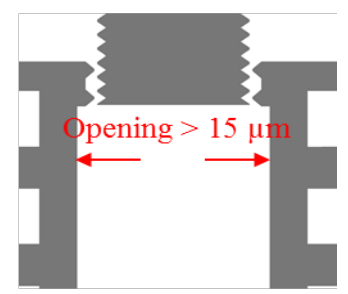

(b)

Fig. 8. Zoom on the contact zone between the moving part and S2 clamp when. A) the clamp holds the moving part. b) it releases the moving part. 


\subsection{Subsystem S3}

As for subsystem S3, it allows closing S3 clamp using actuators in order to hold the moving part during stepping of the multistable actuator. It consists of two U-shaped electrothermal actuators and S3 clamp which is a set of two gantries as shown in Fig. 9.

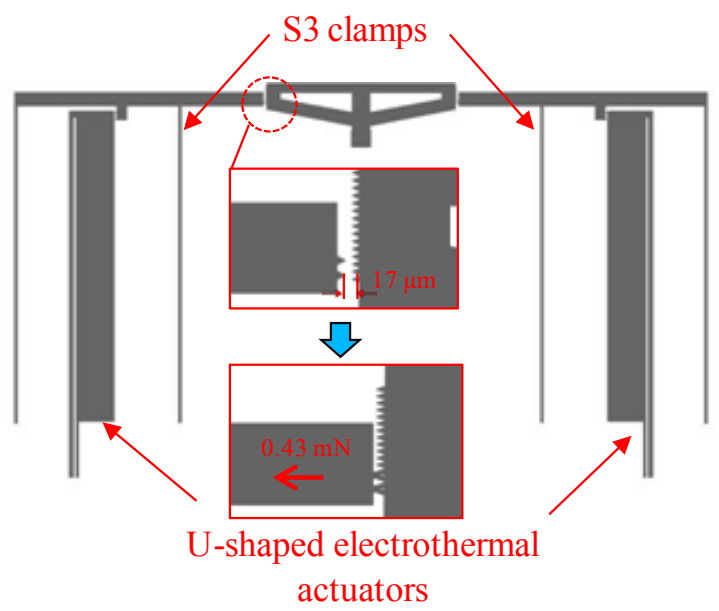

Fig. 9. Drawing of subsystem S3, including a zoom on the teeth configuration at rest.

The activation phase does not concern subsystem S3 where S3 clamp is normally open. The horizontal distance between the open and closed configurations for each gantry of S3 clamp is $17 \mu \mathrm{m}$. This distance and the stiffness of the S3 gantry determine the elastic force, $0.43 \mathrm{mN}$, that the electrothermal actuator have to overcome for closing/engaging the S3 clamp between the teeth of the moving part.

\subsection{Moving part}

As for the moving part, its butterfly shape allows reducing the horizontal extension of the gantries. A couple of curved beams are used in order to maintain the moving part and to guide a vertical displacement as shown in fig. 10.

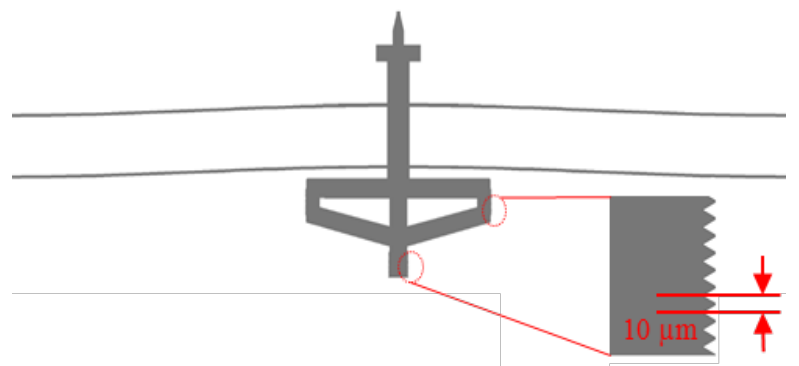

Fig. 10. Moving part of the multistable actuator connected to curve beams.

The dimensions of the curved beams are as follows $(1=6.8 \mathrm{~mm}, \mathrm{~h}=60 \mu \mathrm{m}, \mathrm{t}=15 \mu \mathrm{m}, \mathrm{b}=100$ $\mu \mathrm{m})$. These dimensions are chosen to define the stroke of the moving part and to reduce the snapping forces as possible. The stroke of the moving part is equivalent to $120 \mu \mathrm{m}$ decomposed in 12 steps of $10 \mu \mathrm{m}$ and 13 stable positions. Thus, the teeth are designed to have 13 engaged positions with the clamps. The as-fabricated curved beams form is buckled upwards, the as-fabricated position of the moving part is the initial position while the other 
positions are in the downward direction. Fig. 11 shows the evolution of the snapping forces of the curved beams during deflection and after each step.

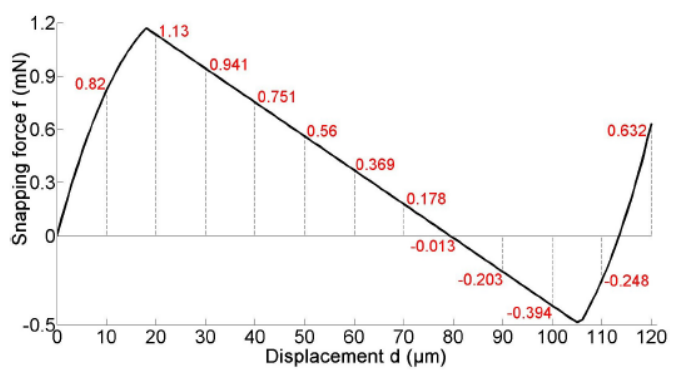

Fig. 11. Evolution of the snapping forces of the curved beams connected to the moving part after deflection and their values at each stable position.

Positive values of the snapping forces in Fig. 11 are helpful when the actuators in subsystem $\mathrm{S} 1$ are pushing the moving part upwards, and have an opposite contribution when the curved beams of subsystem S1 are pushing the moving part downwards. Thus, the greatest loads on the actuators of subsystem S1 exists during transition between 100 and $110 \mu \mathrm{m}$ while the lowest driving force of the curved beams of subsystem S1 in the downward direction is between 10 and $20 \mu \mathrm{m}$.

\subsection{U-shaped electrothermal actuators}

In each subsystem of discrete actuator, U-shaped electrothermal actuators are used in order to ensure the switching function (closing and opening of S2 and S3 clamp) and to drive and hold the moving part in the second stable position (shuttle in subsystem S1). The dimensions of the U-shaped electrothermal actuator are chosen based on the modeling and FEM simulations [37] in order to provide the required performance in terms of force and displacement. The final dimensions of the actuators are shown in figure 12.

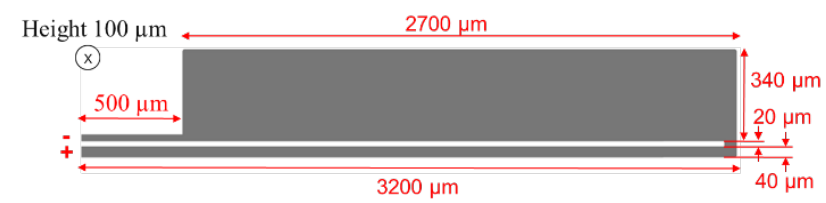

Fig. 12. Dimensions of the U-shaped electrothermal actuator.

Under voltage of $20 \mathrm{~V}$, the electrothermal actuator can reach a displacement of $200 \mu \mathrm{m}$, after $80 \mathrm{~ms}$, generating a force higher than $10 \mathrm{mN}$.

Figure 13 shows a drawing of a prototype of the discrete actuator. The total planar dimension of the discrete actuator is equivalent to $12 * 11 \mathrm{~mm}$ including the support, conductive lines and the active parts.

The different electrothermal actuators are connected electrically to the pads at the bottom edge of the discrete actuator through conductive lines in the gold layer as shown in Fig. 13. Each conductive line with its correspondent pad is isolated electrically from the device by 
etching their borders. One other pad is deposited in the middle between the other pads in order to impose the electrical potential of the device.

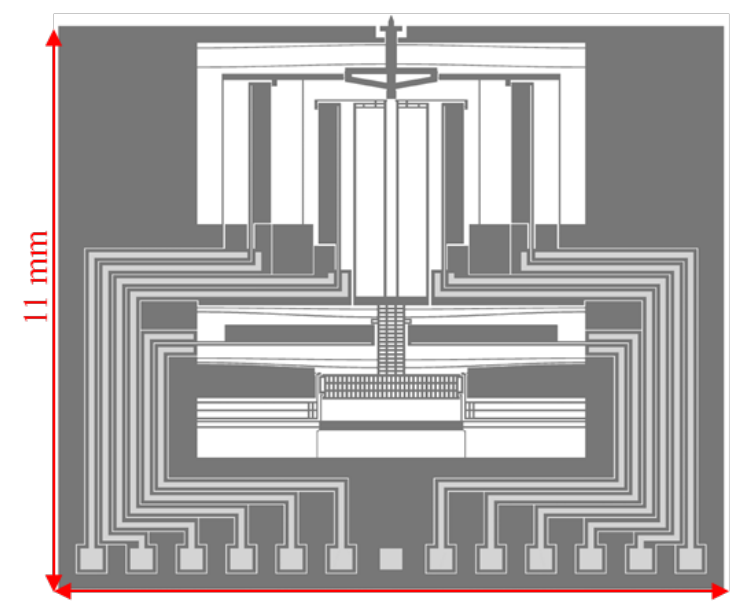

$12 \mathrm{~mm}$

Fig. 13. Drawing of the discrete actuator including all subsystems, the support and conductive lines.

\section{Microfabrication}

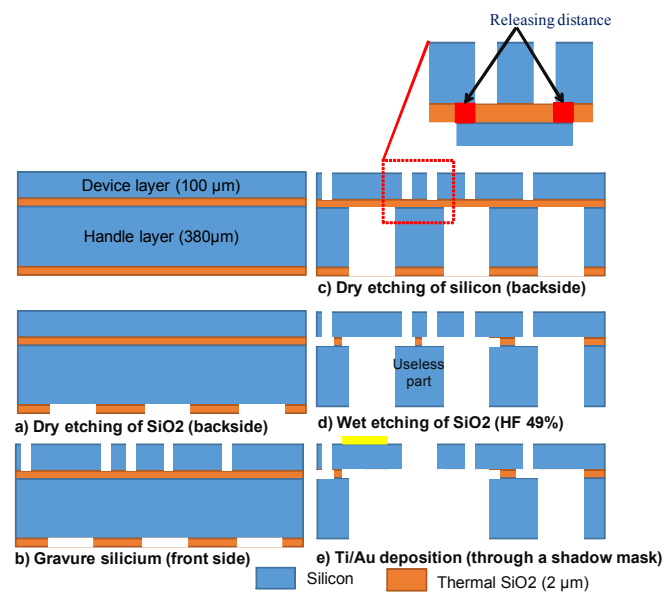

Fig. 14. Fabrication process steps of the multistable actuator on SOI wafers.

The multistable actuator in this study was fabricated using $<100>$ oriented and highly doped p-type single-crystalline silicon-on-insulator (SOI) wafer, the corresponding electrical resistivity is $0.01-0.03 \mathrm{ohm} . \mathrm{cm}$. The SOI wafer is composed of $100 \mu \mathrm{m}$ thick silicon device layer, dedicated to the active area, a $2 \mu \mathrm{m}$ thick buried silicon dioxide ( $\mathrm{SiO} 2)$ separates this device layer from $380 \mu \mathrm{m}$ thick handle silicon layer. The backside of the SOI wafer consists of $2 \mu \mathrm{m}$ thick silicon dioxide layer, which serves as a hard mask during the silicon etching step.

First, the backside silicon dioxide layer is patterned and dry etched using reactive ion etching RIE process. The opening windows are $60 \mu \mathrm{m}$ width, in order to get a homogeneous profile during the silicon dry etching on the backside (Fig. 14a). 
The topside, device layer, is lithographically patterned with opening widths of $20 \mu \mathrm{m}$ and dry etched using deep reactive ion etching DRIE process (Fig. 14b), followed by the dry etching of the handle layer using DRIE process (Fig. 14c). After etching, the wafer is dived into HF solution (49\%) in order to release the useless parts from the wafer (Fig. 14d). The HF solution etched transversally the buried silicon dioxide located between the device and handle openings. The releasing distance is $30 \mu \mathrm{m}$. Finally, the last step consists of depositing a gold layer of 200nm thick through a shadow mask (Fig. 14e).

Figure 15 shows an optical image of the multistable actuator after fabrication.

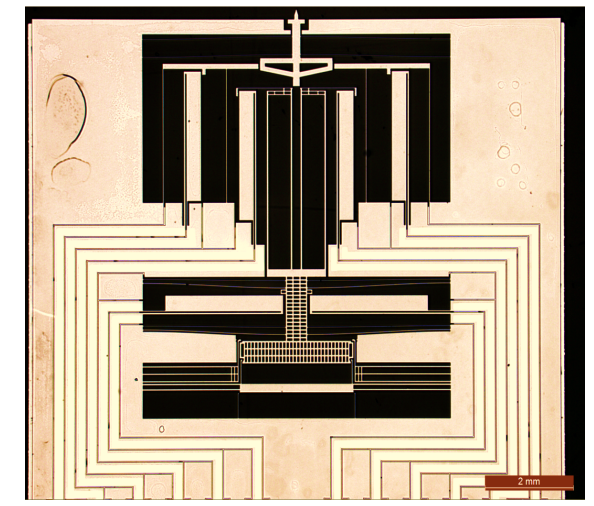

Fig.15. Optical image of multistable actuator.

After fabrication, the multistable actuator requires an activation step. It consists in arming, manually using probe head, the different components in the mechanical stop mechanism in subsystem S1(Fig16-1 b to Fig16-2 b) and arming the teeth between subsystem 2 latch and the moving part (Fig16-1 a to Fig 16-2 a). During this manual phase, first probeheads are used to open S2 clamp (fig. 16-1a), second probeheads are used to set the upper stop at the upper lock (fig. 16-1b, down right red arrow), and third probeheads are used to simultaneously set the lower stop at the lower lock (fig. 16-1b, down middle red arrow) and the S2 clamp at the lower stop (fig. 16-1b, up left red arrow). The operation described in the fig. 16-1b leads to the configuration of the fig. 16-2b and consequenty to the vertical displacement of the S2 clamp facing the teeth of moving part. The releasing of the S2 clamp by the first probeheads provides the teeth engagement between the S2 clamp and the moving part as described by the fig. 16-2a.

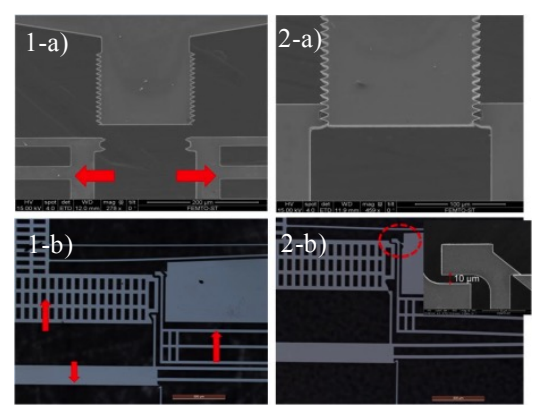

Fig. 16. Optical pictures showing: 1) Before activation: a) system 2 with moving part, b) mechanical stop mechanism, 2) after activation steps of the multistable actuator. 
After activation, the multistable actuator is glued on a PCB card. A wire bonding is performed in order to connect the device pads to the PCB pads.

\section{Experimental characterization}

To supply the different actuators, the PCB with the multistable actuator are connected to an electronic circuit. It consists of a microcontroller to control the supply time and sequence order for supplying the different U-shaped electrothermal actuators, connectors and a relay circuit to switch on or off the current at each single electrothermal actuator stage. Buttons allow the switch control between upward and downward steps.

Chronograms of U-shaped electrothermal actuators of each subsystem are shown for upward and downward steps in fig. 17. A supply time of $20 \mathrm{~ms}$ is chosen for switching each actuator to their activated state. In the other side, a time of $10 \mathrm{~ms}$ is chosen when releasing each subsystem to return to its initial state elastically.

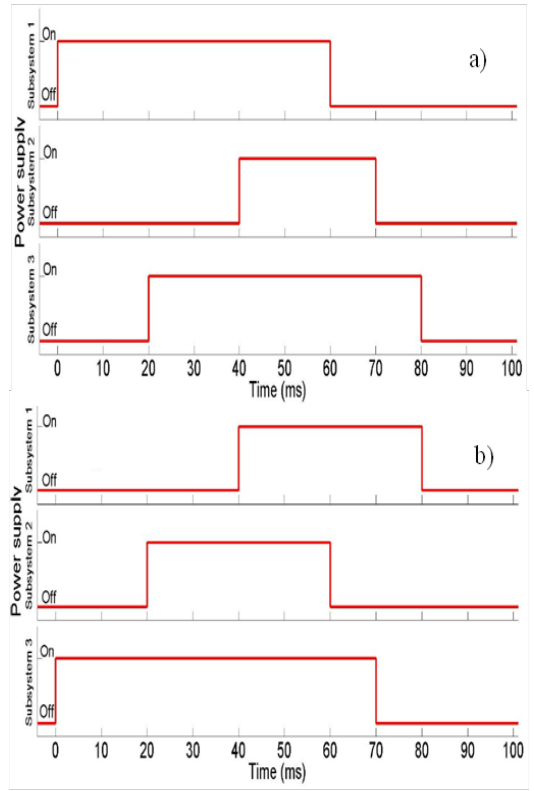

Fig. 17. Sequence order and supply times for the electrothermal actuators of subsystem S1,S 2 and S3 in order to make: a) an upward step, b) a downward step.

In order to validate the correct operation of designed prototype, the sequence order shown in Fig. 17 has been applied to the multistable actuator. The desired mechanical task has been achieved successfully by the multistable actuator. Figure 18 shows the device response to make a downward step recorded using the high-speed camera. 


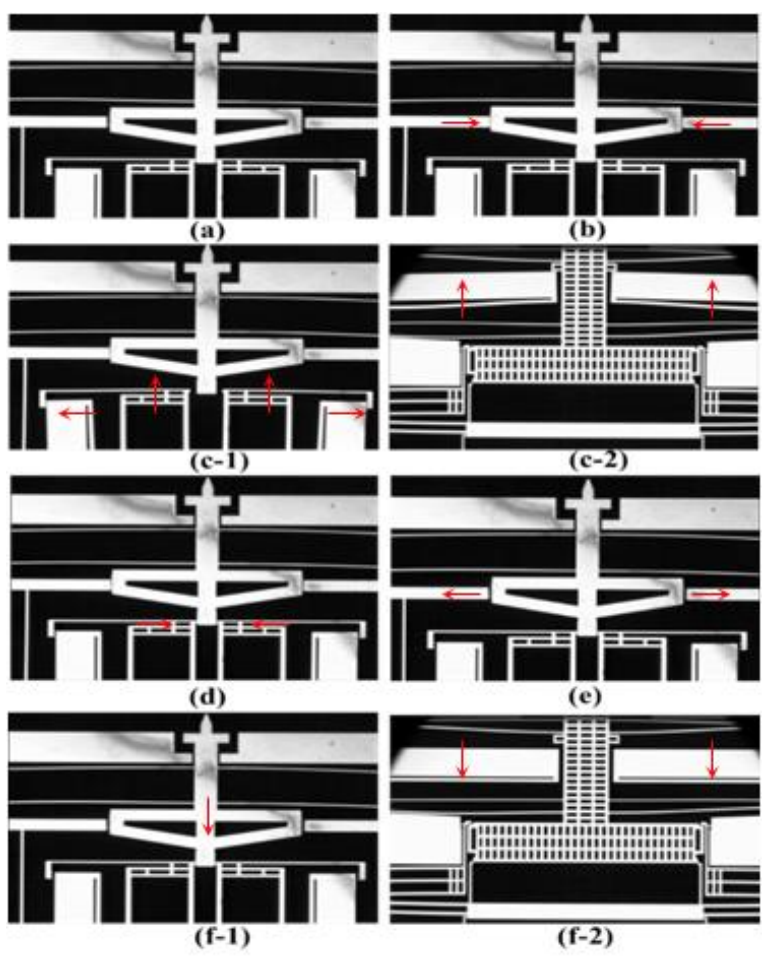

Fig. 18. Experimental sequence order to make a downward step: b) S3 clamp holds the moving part, c1\&2) S2 clamp releases the moving part and moves upwards thanks to S1, d) S2 closes in the upper position, e) S3 clamp releases then the moving part, f-1\&2) S2 clamp moves downwards with holding the moving part thanks to $\mathrm{S} 1(\mathrm{f})$.

This simple task allows us to validate the operating principal of fabricated devices and the suitability of the design.

Figure 19 shows the S2 clamp of subsystem S2 on the moving part at the initial position and after 10 downward steps.

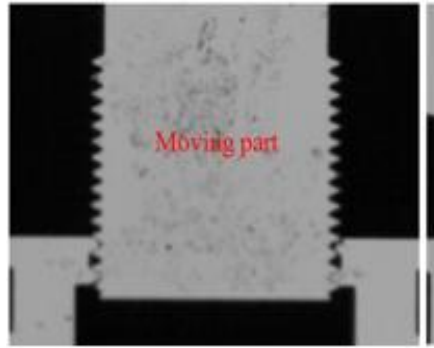

(a)

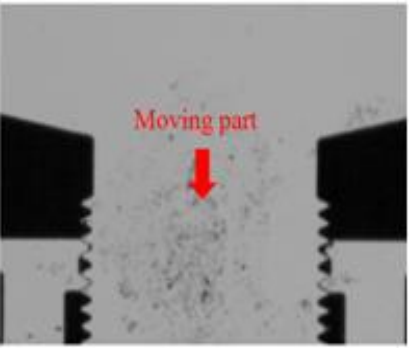

(b)

Fig. 19. Photos of subsystem S2: a) at the initial position, b) after 10 downward steps.

In order to quantitatively measure the generated displacement of the multistable actuator, 12 upward steps followed by 12 downward steps are applied to the device. The corresponding response is recorded thanks to the Micro System Analyzer MSA-500 from Polytec, which can measure nanometric size displacements, in an ambient environment, using the Planar Motion Analysis software. The obtained results representing the achieved steps are reported in Fig. 20 and table 1. 


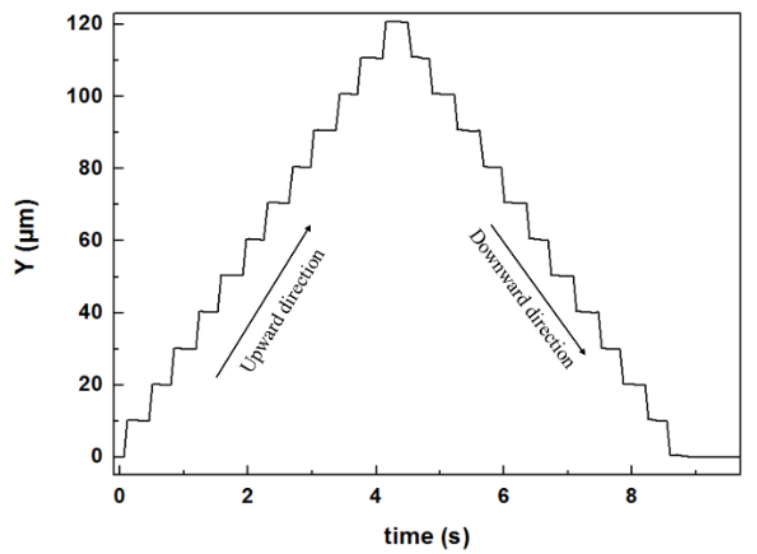

Fig. 20. Positions of the multistable actuator reached during 12 upward steps followed by 12 downward steps.

Table 1. Extracted positions of the multistable actuator during 12 upward steps followed by 12 downward steps.

\begin{tabular}{cc}
\hline Position number & Position $(\mu \mathrm{m})$ \\
\hline 1 & $0.01 \pm 0.08$ \\
2 & $10.12 \pm 0.07$ \\
3 & $20.08 \pm 0.13$ \\
4 & $30.15 \pm 0.14$ \\
5 & $40.24 \pm 0.09$ \\
6 & $50.33 \pm 0.10$ \\
7 & $60.31 \pm 0.10$ \\
8 & $70.51 \pm 0.10$ \\
9 & $80.49 \pm 0.09$ \\
10 & $90.55 \pm 0.11$ \\
11 & $100.64 \pm 0.12$ \\
12 & $110.66 \pm 0.12$ \\
13 & $120.67 \pm 0.08$ \\
\hline
\end{tabular}

According to obtained results, the mean achieved displacement is $120.67 \pm 0.08 \mu \mathrm{m}$ over 12 upward steps with a mean step of $10.06 \pm 0.09 \mu \mathrm{m}$. Knowing that the theoretical step is fixed to $10 \mu \mathrm{m}$, the maximal displacement is expected to be $120 \mu \mathrm{m}$. This small variation between theoretical and experimental displacement (max error of $\pm 1.4 \%$ ) can be explained by the tolerances resulting from the fabrication process. Indeed, the distance between two successive teeth in the moving part, $10 \mu \mathrm{m}$, is not equal to the shuttle displacement in the subsystem S1 $(10.2 \mu \mathrm{m})$. This difference causes a backlash (bad teeth engagement) between the teeth of S2 clamps and moving part teeth after an up/downward displacement. The resulted backlash may generate randomly a slipping or not of the clamps teeth on the moving part teeth, thanks to the horizontal restoring force of S2 clamp.

These first experimental results show a promising application of the multistable actuator for microrobotic task. Indeed, it can be used for accurate positioning applications in MEMS or integrated in more complex systems for more advanced tasks. 


\section{Conclusion}

A multistable actuator is presented in this paper. It allows switching the moving part between several stable positions linearly in a one-dimensional direction back and forth. The presented discrete acruator combines advantages of digital microrobotics, monolithic structures, compliant and unlimited multistable mechanisms.

The principle of multi-stability is based on a specific sequence order of moving and opening a normally closed microgripper and closing a normally open microgripper. The microgrippers hold the moving part in the stable positions during operation and at rest. The design of the discrete actuator, the fabrication process and experimental results are presented in the paper. The studied prototypes showed a proper operation. The mean achieved displacement was $120.67 \pm 0.08 \mu \mathrm{m}$ over 12 upward steps with a mean step of $10.06 \pm 0.09 \mu \mathrm{m}$. A max error of $\pm 1.4 \%$ was noticed on achieved position during upward and downward direction steps. It was settled that this difference is caused by the microfabrication tolerance. However these performances allow making tasks in MEMS applications.

Significant prospects for developing the discrete actuator are possible in future works including the following topics: optimizing and reducing the total sequence time needed for each step, testing under loaded condition, integrating the discrete actuator in more complex systems and developing the principle of the mechanism towards making planar discrete positioning.

\section{Acknowledgement}

This work has been supported by the Labex ACTION project (contract ANR-11-LABX-0101), the French RENATECH network and its FEMTO-ST technological facilities.

\section{References}

[1] Q. Chen, Y. Haddab, and P. Lutz, "Digital Microrobotics Based on Bistable Modules: Design of Compliant Bistable Structures," in 2008 IEEE/ASME International Conference on Mechtronic and Embedded Systems and Applications, 2008, pp. 36-41.

[2] P. Cazottes, A. Fernandes, J. Pouget, and M. Hafez, "Actuation of bistable buckled beams with Macro-Fiber Composites," in 2008 IEEE/RSJ International Conference on Intelligent Robots and Systems, 2008, pp. 564-569.

[3] V. Chalvet, Y. Haddab, and P. Lutz, "A Microfabricated Planar Digital Microrobot for Precise Positioning Based on Bistable Modules," IEEE Trans. Robot., vol. 29, no. 3, pp. 641-649, Jun. 2013.

[4] D.-A. Wang, H.-T. Pham, and Y.-H. Hsieh, "Dynamical switching of an electromagnetically driven compliant bistable mechanism," Sens. Actuators Phys., vol. 149, no. 1, pp. 143-151, Jan. 2009.

[5] H. Ren and E. Gerhard, "Design and fabrication of a current-pulse-excited bistable magnetic microactuator," Sens. Actuators Phys., vol. 58, no. 3, pp. 259-264, Mar. 1997.

[6] B. D. Jensen, L. L. Howell, and L. G. Salmon, "Design of Two-Link, In-Plane, Bistable Compliant Micro-Mechanisms," J. Mech. Des., vol. 121, no. 3, pp. 416-423, Sep. 1999.

[7] N. D. Masters and L. L. Howell, "A self-retracting fully compliant bistable micromechanism," $J$. Microelectromechanical Syst., vol. 12, no. 3, pp. 273-280, Jun. 2003.

[8] M. Freudenreich, U. Mescheder, and G. Somogyi, "Simulation and realization of a novel micromechanical bi-stable switch," Sens. Actuators Phys., vol. 114, no. 2-3, pp. 451-459, Sep. 2004. 
[9] D. L. Wilcox and L. L. Howell, "Fully compliant tensural bistable micromechanisms (FTBM)," J. Microelectromechanical Syst., vol. 14, no. 6, pp. 1223-1235, Dec. 2005.

[10] J. Qiu, J. H. Lang, A. H. Slocum, and A. C. Weber, "A bulk-micromachined bistable relay with U-shaped thermal actuators," J. Microelectromechanical Syst., vol. 14, no. 5, pp. 1099-1109, Oct. 2005.

[11] Y. Zhang, G. Ding, X. Shun, D. Gu, B. Cai, and Z. Lai, "Preparing of a high speed bistable electromagnetic RF MEMS switch," Sens. Actuators Phys., vol. 134, no. 2, pp. 532-537, Mar. 2007.

[12] Y. Wu, G. Ding, C. Zhang, J. Wang, S. Mao, and H. Wang, "Design and implementation of a bistable microcantilever actuator for magnetostatic latching relay," Microelectron. J., vol. 41, no. 6, pp. 325-330, Jun. 2010.

[13] J. Barth and M. Kohl, "A bistable magnetically enhanced shape memory microactuator with high blocking forces," Phys. Procedia, vol. 10, pp. 189-196, Jan. 2010.

[14] Z. Yu et al., "Bistable electroactive polymers (BSEP): large-strain actuation of rigid polymers," 2010, vol. 7642, p. 76420C-76420C-9.

[15] S. Park and D. Hah, "Pre-shaped buckled-beam actuators: Theory and experiments," Sens. Actuators Phys., vol. 148, no. 1, pp. 186-192, Nov. 2008.

[16] Q. Chen, Y. Haddab, and P. Lutz, "Microfabricated bistable module for digital microrobotics," $J$. Micro-Nano Mechatron., vol. 6, no. 1-2, pp. 1-12, Feb. 2011.

[17] H. W. Huang and Y. J. Yang, "A MEMS Bistable Device With Push-On \#x2013;Push-Off Capability," J. Microelectromechanical Syst., vol. 22, no. 1, pp. 7-9, Feb. 2013.

[18] D. W. Pohl, "Dynamic piezoelectric translation devices", Rev. Sci. Instum.,vol. 58, pp. 54-57, 1987.

[19] K. Besocke, "An easily operable scanning tunneling microscope”, Surface Science, vol. 181, pp. 145-153, 1987.

[20] L. Howald, H. Rudin et H.-J. Güntherodt, “ Piezoelectric inertial stepping motor with spherical rotor”, Rev. Sci. Instrum., vol. 63, pp. 3909-3912, 1992.

[21] J.-M. Breguet et P. Renaud, “A 4-degrees of freedom microrobot with nanometer resolution”, Robotica, vol. 14, pp. 199-203, 1996.

[22] V. Guyenot, C. Siebenhaar et C. Damm, "A kicking step by step drive for precision engineering”, 8th IPES, Compiègne, France, pp. 278-281, mai 1995.

[23] R. Matsuda et R. Kaneko, "Micro-step XY-stage using piezoelectric tube actuator”, MEMS'91, NARA, JAPAN, pp. 137-142, 30 janvier - 2 février 1991.

[24] R. Büchi, W. Zesch, A. Codourey et R. Siegwart, "Inertial drives for micro- and nanorobots: Analytical study", SPIE's, Intelligent Systems and Advanced Manufacturing, Philadelphia, USA, 23-26 octobre 1995.

[25] R. C. Roth, "The elastic wave motor a versatile terfonol driven, linear actuator with high force and great precision", DynaMotive Corporation, Richmond, Canada, 1990.

[26] M. Bexell et S. Johanson, "A high torque miniature inchworm motor", Int. Conf. on Solid-State Sensors and Actuators Eurosensors IX, pp. 69-70, juin 1995.

[27] J. Oberhammer, M. Tang, A.-Q. Liu, and G. Stemme, "Mechanically tri-stable, true single-poledouble-throw (SPDT) switches," J. Micromechanics Microengineering, vol. 16, no. 11, p. 2251, 2006.

[28] G. Chen, D. L. Wilcox, and L. L. Howell, "Fully compliant double tensural tristable micromechanisms (DTTM)," J. Micromechanics Microengineering, vol. 19, no. 2, p. 25011 , 2009.

[29] G. Chen, Q. T. Aten, S. Zirbel, B. D. Jensen, and L. L. Howell, "A Tristable Mechanism Configuration Employing Orthogonal Compliant Mechanisms," J. Mech. Robot., vol. 2, no. 1, pp. 14501-14501-6, Nov. 2009.

[30] D.-A. Wang, J.-H. Chen, and H.-T. Pham, "A tristable compliant micromechanism with two serially connected bistable mechanisms," Mech. Mach. Theory, vol. 71, pp. 27-39, Jan. 2014. 
[31] J. Zhao, R. Gao, G. Chen, S. Liu, Q. Cao, and T. Qiu, "Nonlinear coupling mechanical model for large stroke magnetic-based multistable mechanisms," Mech. Mach. Theory, vol. 83, pp. 56-68, Jan. 2015.

[32] J. S. Han, C. Müller, U. Wallrabe, and J. G. Korvink, "Design, Simulation, and Fabrication of a Quadstable Monolithic Mechanism With X- and Y-Directional Bistable Curved Beams," $J$. Mech. Des., vol. 129, no. 11, pp. 1198-1203, Nov. 2006.

[33] H.-T. Pham and D.-A. Wang, "A quadristable compliant mechanism with a bistable structure embedded in a surrounding beam structure," Sens. Actuators Phys., vol. 167, no. 2, pp. 438-448, Jun. 2011.

[34] Y. Gerson, S. Krylov, B. Ilic, and D. Schreiber, "Design considerations of a large-displacement multistable micro actuator with serially connected bistable elements," Finite Elem. Anal. Des., vol. 49, no. 1, pp. 58-69, Feb. 2012.

[35] H. Hussein, P. Le Moal, G. Bourbon, Y. Haddab, and P. Lutz, "Modeling and stress analysis of a pre-shaped curved beam: influence of high modes of buckling," International Journal of Applied Mechanics, vol. 7, no. 04, p. 1550055, 2015.

[36] H. Hussein, G. Bourbon, P. L. Moal, Y. Haddab, and P. Lutz, "Mechanical stop mechanism for overcoming MEMS fabrication tolerances," J. Micromechanics Microengineering, vol. 27, no. 1, p. 17001, 2017.

[37] H. Hussein, A. Tahhan, P. L. Moal, G. Bourbon, Y. Haddab, and P. Lutz, "Dynamic electrothermo-mechanical modelling of a U-shaped electro-thermal actuator," J. Micromechanics Microengineering, vol. 26, no. 2, p. 25010, 2016. 